\title{
On the Statistical Theory of Electromagnetic Waves in a Fluctuating Medium (II)
}

\section{Mathematical Basis of the Analogies to Quantum Field Theory (a Digest)}

\author{
K. Furutsu* \\ Central Radio Propagation Laboratory, National Bureau of Standards, Boulder, Colo.
}

(Received June 23, 1965)

\begin{abstract}
Many analogies to quantum field theory exist inherently in the statistical theory of waves; for instance, the problem of getting the effective medium constant and the effective coupling constant between the waves and the fluctuating medium can be treated in almost the same way as in field theory (theory of renormalization). These analogies are shown to be due to the fact that basic equations exist in the statistical theory which correspond closely to the fundamental equations in field theory; i.e., to the commutation relations and the Heisenberg equation of motion. A probability density function of waves is introduced here which corresponds to the probability amplitude function in quantum mechanics. In this case, the boundary conditions at infinity for this probability density function are found to be expressed in the same form as the vacuum boundary conditions in field theory. The theory of the statistical Green's functions and their relationships to the expectation values of physical variables is also extensively developed, using auxiliary external sources of the wave and of the fluctuating medium. It is found that there exists a one-to-one correspondence between the formalism of Green's functions presented here and that used in field theory. The above correspondence may be important for the further development of the statistical theory of waves, just as the advanced techniques of field theory have greatly influenced the development of thermodynamics.

The details of the theory were recently published [Furutsu, 1965].
\end{abstract}

In recent years, important advances have been carried out in the field of statistical physics by the successful application of the well developed methods of quantum field theory to the study of systems consisting of a large number of interacting particles which are in thermodynamic equilibrium [Matsubara, 1955; Martin and Schwinger, 1959; Alekseev, 1961]. In particular, the method of thermodynamic Green's functions has been extensively used in the study of important problems of statistical physics and, in principle, all the necessary physical observables seem to have been obtained in terms of the many particle Green's functions.

The latter situation is the same also in the statistical theory of ordinary waves in a fluctuating medium, as is emphasized in part I of this series of papers [Furutsu, 1963], ${ }^{1}$ and all the necessary statistical information about the system of waves can be obtained in terms of the (statistical) Green's functions of various orders. On the other hand, particularly when the medium fluctuation follows the Gaussian multivariate

*Present address, Radio Research Laboratories, Kokubunji, Kitatama-Gun, Tokyo, Japan.

${ }^{1}$ Recently, R. C. Bourret notified the author of his recent paper [1962] in which he also suggested a strong correspondence with field theory by several examples. normal distribution, the Green's functions can be derived in any order of approximation by using the Feynman diagram technique in field theory; thus the corresponding graphs may be divided into several parts such as the self-energy parts and the vertex parts, and the same techniques as used in field theory may be available to treat these parts of the graphs (theory of renormalization). ${ }^{2}$

Here, it may be noticed that the diagram technique in field theory is a due consequence of the quantization of a field, the Heisenberg equation of motion, and the boundary (vacuum) condition at infinity. Thus, if the expectation values of all the physical observables in field theory are to be obtained in terms of Green's functions, we may ask the question whether or not it is possible, starting with the knowledge of the Green's functions, to derive, inversely, the commutation relations and the equation of motion, which are the fundamental basis of quantum field theory.

The major purpose of a recent paper [Furutsu, 1965] is to prove the existence, in the statistical theory of waves, of fundamental equations correspond-

${ }^{2}$ A similar diagram method is adopted in the theory of scattering by impurities in metals by Rickayzen [1961]. 
ing to the quantum mechanical commutation relations between the physical variables involved and also the Schrödinger equation (or the Heisenberg equation of motion in the Heisenberg picture) together with the boundary condition at infinity corresponding to the vacuum condition. As the due consequence of this correspondence, many analogies to the field theory, particularly in the formulation of the Green's functions, follow. The knowledge of this fundamental relationship between the two theories may be worthwhile, since other useful techniques used in the field theory, such as unperturbational methods not based on Born series may be available also in the statistical theory of waves.

(Paper 69D11-582)

\section{References}

Alekseev, A. I. (1961), The application of the methods of quantum field theory in statistical physics, Soviet Phys. Uspekhi 4, 23.

Bourret, R. C. (1962), Stochastically perturbed fields, with applications to wave propagation in random media, Nuovo Cimento 26, 1 .

Furutsu, K. (1963), On the statistical theory of electromagnetic waves in a fluctuating medium (I), J. Res. NBS 67D (Radio Prop.), No. 3, 303 .

Furutsu, K. (1965), On the statistical theory of electromagnetic waves in a fluctuating medium (II), Mathematical basis of the analogies to quantum field theory, NBS Mono. 79.

Matsubara, T. (1955), A new approach to quantum-statistical mechanics, Prog. Theoret. Phys. 14, 351.

Martin, P. C., and J. Schwinger (1959), Theory of many-particle systems (I), Phys. Rev. 115, 1342.

Rickayzen, G. (1961), Scattering by impurities in metals, "Manybody problem" (W. A. Benjamin, Inc., New York, N.Y.). 


\section{Reviews and Selected Abstracts in Radio Science*}

\begin{abstract}
Plane-wave spectrum representation of a field in a magnetionic medium due to a plane distribution of source currents, C. Bates and R. Mittra, Electronics Letters 1, No. 3, 74 (May 1965).

The fields due to a plane distribution of source currents are formulated, in terms of three-dimensional Fourier transforms, to yield equivalent results obtainable from a representation in terms of an angular spectrum of plane waves introduced by Clemmow. Certain difference in the present field expressions and those given by Clemmow in a recent paper are discussed.
\end{abstract}

Radar backscatter from the surface of the moon, P. Beckmann, J. Geophys. Res. 70, No. 10, 2345-2350 (May 15, 1965). The experimentally measured dependence of the mean radar power backscattered from the lunar surface on the angle of incidence (delay time) is compared with the theoretical formula based on a lunar surface consisting of a superposition of several normal random processes of different structures. The theoretically derived curve accounts for shadowing and matches the experimental data over almost the entire range of angles of incidence to within $\pm 1 \mathrm{db}$. The wavelength dependence is also consistent with the observed data as far as they are available. The analysis yields a value of $11^{\circ}$ for the rms slope of the lunar surface.

Coupled-modes analysis of the phonon-photon parametric backward-wave oscillator, D. L. Bobroff, J. Appl. Phys. 36, No. 5, 1760-1769 (May 196.5).

Because of the phenomenon of electrostriction, the passage of an intense laser beam through a medium can lead to a parametric backward-wave interaction in which one of the two waves (signal and idler) is an acoustic wave and the other an optical wave. This paper analyzes this effect and determines the conditions necessary for backward-wave oscillations to occur. The time rate of build-up of the oscillations when these conditions are satisfied is determined. The behavior of the system as an amplifier of acoustical and optical energy is also determined.

Radio wave emissions in the v.l.f.-band observed near the auroral zone-I. Occurrence of emissions during disturbances, L. Harang and R. Larsen, J. Atmospheric Terrest. Phys. 27, No. 4, 481-497 (Apr. 1965).

Very low frequency emissions in the $8 \mathrm{kc} / \mathrm{s}$-band have been recorded at the Auroral Observatory, Tromsö and bursts of enhanced emissions have been compared with geomagnetic records, absorption effects recorded on a riometer and appearance of aurorae. It is shown that bursts of enhanced radiations in the $8 \mathrm{kc} / \mathrm{s}$-band appear regularly during disturbed conditions near the auroral zone, the energy is of the order $1-10 \times 10^{-16} \mathrm{~W} / \mathrm{m}^{2} \mathrm{c}$. The occurrence of the bursts exhibits a pronounced diurnal variation, their occurrence is limited to the time interval 1800-2400 hours M.E.T. The average duration of the bursts may vary from one minute to several hours, with a maximum occurrence of $10-12 \mathrm{~min}$ duration. The v.l.f.emissions show a complicated dependence on geomagnetic disturbances and absorption within the ionosphere. Small dips of absorption in the riometer curves of the order $0 \cdot 3-0.9 \mathrm{~dB}$ may by accompanied by v.l.f.-emmissions; there is a positive correlation. Great values of absorption of the order $5-9 \mathrm{~dB}$ are accompanied by a complete cessation of v.l.f.-emissions; there is a negative correlation. These two effects indicate conclusively that the v.l.f.-emissions are strongly absorbed in a disturbed ionosphere. The moderate positive geomagnetic disturbances in $H$ appearing early in the night (which is often followed by quiet auroral forms, ares and bands on the Northern sky) are very often accompanied by strong v.l.f.-emissions. Sudden and strong disturbances in the geomagnetic field when these appear as a sudden commencement, are usually followed by a pronounced v.l.f.-emission. When the disturbance develops with (usually negative) peaks in $H$ the v.l.f.-emissions are completely absorbed. It is pointed out that the close connection between the v.l.f.-emissions and the geomagnetic disturbances is limited to appear only in the time interval 1800-2400 hours M.E.T. Outside this 6-hr interval v.l.f.-emissions do not appear independently of the degree of geomagnetic disturbances, auroral luminosities or level of ionospheric absorption. The point of view that the v.l.f.-emissions are generated in the near and local part of the ionosphere by an impact of charged particles (for instance as Cerenkov-radiation) which simultaneously are producing auroral luminosities, must therefore be regarded as less probable. If this were the case one would expect an appearance of v.l.f.-emissions throughout the whole night. Records demonstrating the parallel amplitude variations in v.l.f.-emissions and 5577 A-photometer recordings are shown. Parallel records of v.l.f. emissions have been made at Troms $\ddot{~}$ and Kiruna, the latter lying $210 \mathrm{~km}$ to the South of Tromsio. In some cases the Kiruna-records show similar changes in amplitudes for great bursts as the Tromsörecords. Three cases of "noise storms" have been recorded during which the enhanced v.l.f.-radiations appeared during the whole night, the "noise storms" coinciding with large geomagnetic disturbances. The amplitude of the "storm" declined and disappeared when the ionosphere was illuminated by the sun's rays in the morning and when the normal day-time absorption was formed. In an Appendix several cases of enhanced radiation are presented on the riometer recording frequency of $28 \mathrm{Mc} / \mathrm{s}$. Records are shown which demonstrate coincidence between v.l.f.-bursts on $8 \mathrm{kc} / \mathrm{s}$ and sudden increases in the normal galactic noise level received on $28 \mathrm{Mc} / \mathrm{s}$. These common bursts appear only when a sudden deflection in the geomagnetic curves appears, which may often have the character of a sudden commencement. The $28 \mathrm{Mc} / \mathrm{s}$ bursts are rare events, but it is concluded that the bursts are not produced by man-made interference.

Radio wave emissions in the v.l.f.-band observed near the auroral zone-II. The physical properties of the emissions, L. Harang and K. N. Hauge, J. Atmospheric Terrest. Phys. 27, No. 4, 499-512 (Apr. 1965).

The direction of arrival of the v.l.f.-emission in the $8 \mathrm{kc} / \mathrm{s}$ bands has been studied using two crossed loops with their planes lying in EW and NS directions, and by switching the loops alternately to the same amplifier channel. The v.l.f.-noise level was higher in the EW than in the NS direction, and this higher EW noise level was maintained during day and night for a year and also independently at two observing places lying $35 \mathrm{~km}$ apart. The v.l.f.-bursts came in on both frames with amplitudes of the same order of magnitude. It is concluded that Tromsö is lying within an area of arrival of the v.l.f.emissions, and that the energy is coming in from all directions. This point of view is confirmed by the polarization studies. The v.l.f.-emissions were picked up on two crossed frames, each of which was connected to identical amplifiers and displayed on the pairs of $X, Y$-plates on an oscillograph. Ordinary broad and irregular v.l.f.-bursts showed an irregular oscillograph pattern with changing amplitudes and irregularly appearing reversals of the sense of rotation. The oscillograph patterns are to be explained by the simultaneous arrival of several v.l.f.-noise trains which are uncorrelated. In some few cases when a sudden and single, sharp burst appeared the oscillograph pattern was different, the pattern was almost circular and the sense of rotation was always in the same direction. In this case it is assumed that the emission came down vertically along the field lines, circularly polarized, and only in the extraordinary mode. Examples of amplitude distribution are demonstrated in which a strong v.l.f.-emission is being built up by a series of sudden, discrete trains of bursts which gradually merge together into a continuous emission. The frequency-analysis of a number of bursts in the range $5-13 \mathrm{kc} / \mathrm{s}$ is demonstrated. It seems to be stronger, while at the end of a burst the lower frequency $6-5 \mathrm{kc} / \mathrm{s}$ is the strongest. A possible explanation of this effect is the gradual building-up of an absorption in the E-layer during a disturbance, which in the beginning will influence the higher frequencies more strongly than the lower.

The mechanism of generation of the continuous band-emission. Cerenkov- and gyroradiations, are discussed, and it is concluded that the observations do not give any decisive proof for either of the 
two hypotheses. It is pointed out that the diurnal curve of occurrence of the v.l.f.-emissions at Tromsö (the appearance being limited to the interval 1800-2400 hours M.E.T.) is difficult to explain and some more extended series of observations are needed at various places along the auroral zone.

Influence of collisions on whistler propagation, $\mathrm{P}$. Hedvall and L. Sjögren, Electronics Letters 1, No. 3, 54-55 (May 1965). The dispersion characteristics of whistler waves propagating along a longitudinally magnetized and lossy plasma slab are numerically calculated. It is shown that, if the wavelength of the whistler waves is much smaller than the transverse dimensions of the plasma slab, a nonzero collision frequency can have a large influence on the dispersion characteristics; for such small wavelengths one should expect the whistler dispersion to be close to that of the corresponding plane waves, but this is, for certain parameter values, true only if the collision frequency is assumed to be nonzero.

Microwave impedance of semiconductor posts in waveguides, Part I, R. D. Larrabee, J. Appl. Phys. 36, No. 5, 15971602 (May 1965).

A method for calculating the impedance of a thin inductive post of semiconductor material placed in a waveguide is presented. The calculation has been simplified by treating separately the geometrical and the material aspects of the interaction. The analytical expressions obtained are evaluated numerically in those cases where only two parameters are needed to characterize the semiconductor.

A note on the theory of equatorial sporadic-E, D. K. McIlroy, J. Atmospheric Terrest. Phys. 27, No. 5, 641-643 (May 1965). It is shown how the essential results of Farley's theory of equatorial sporadic- $E$ can be predicted from simple theory and how the simple theory exhibits the physical mechanism underlying Farley's theory. It is concluded however that Farley's more elaborate theory is necessary to predict the observed wavelength dependence of the $E_{s}$ irregularities.

The growth of peak velocity, noise, and signals in O-type electron beams, T. G. Mihran and B. K. Andal, IEEE Trans. Electron Devices ED-12, No. 4, 208-216 (Apr. 1965).

A new phenomenon, the growth of peak velocity of an electron beam originating from a shielded gun, has been investigated. Its behavior with distance, magnetic field, and phasing between beam voltage pulse and heater current zero is presented. This phenomenon bears a general but not a detailed resemblance to the noise growth observed by previous investigators. The noise growth phenomenon has been re-examined in detail on the beam used for peak velocity growth measurements. The single most striking observation is that whereas peak velocity and $3-\mathrm{kmc}$ noise growth take place at magnetic field strength primarily above the Brillouin flow value, 3 -kme signal growth occurs only at magnetic fields below the Brillouin value.

A combination of linear axially symmetric surface-wave mode theory and simple intermodulation theory is unable to describe quantitatively the noise growth rate. Consideration of other axiallysymmetric modes on near-Brillouin beams, namely body modes and near-synchronous modes, also leads to poor correlation with experiment. It seems likely that the correct explanation involves consideration of one or more of the following: 1) theta-varying dc beam media, 2) theta-varying RF excitation, or 3) a more accurate nonlinear representation of noise.

Reduction of electromagnetic backscatter from a plasmaclad conducting body, E. L. Murphy, J. Appl. Phys. 36, No. 6, 1918-1927 (June 1965).

The investigation of dipole resonance effects in electromagnetic scattering from cylindrical plasma columns is currently directed toward understanding the observed multiplicity of resonances and the effect of magnetic fields, finite temperature, glass envelopes, etc., on these resonances. When a conducting core is embedded in the column, the analysis of this paper shows that not only are the shapes and locations of the resonances modified, but in addition, the possibility then exists for a decrease in the backscatter cross section considerably below the value for the conducting core itself. Al- though such plasma-conducting core configurations may not be realizable in the laboratory, large decreases or "dips" in the electromagnetic backscatter from hypersonic projectiles have been observed, and there is the possibility that an ionized cloud surrounding the projectile is responsible for the dip, perhaps through the mechanism described here. In this paper, an analysis of this "resonancedip" phenomenon is described in some detail for the problem of a cylindrical or spherical conducting core surrounded by a uniform concentric plasma layer.

Theory of a pressure-tuned lead salt laser, G. W. Pratt, Jr., and J. E. Ripper, J. Appl. Phys. 36, No. 5, 1525-1527 (May 1965).

The frequency and polarization dependence on applied stress of the output radiation of $\mathrm{PbTe}$ and PbSe diode lasers is discussed. Because the energy gap decreases with applied pressure, the output wavelength can be tuned over a wide range. It is estimated that the emission from a $\mathrm{PbSe}$ diode can be tuned continuously from its unstressed value of $8.5 \mu$ by a 100 uniaxial stress to $9.6 \mu$ at $5 \times 10^{3}$ atm and to $10.5 \mu$ at $10^{4} \mathrm{~atm}$. It is proposed that sound waves can be used to frequency-modulate a laser diode.

Plasma diagnosis by means of optical scattering, S. E. Schwarz, J. Appl. Phys. 36, No. 6, 1836-1841 (June 1965).

When a pulse of intense light from a laser passes through a plasma, a very small fraction of the light is scattered out of the beam by free electrons. Measurements of the intensity and spectral distribution of the scattered light can be related to the electron density and velocity distribution in the plasma. This paper presents a general discussion of the use of optical scattering as a plasma diagnostic technique. Applicability to various experimental situations is discussed, and considerations of interest in the design of diagnostic apparatus are presented.

An apparatus has been constructed for the measurement of electron density in cold plasma. The apparatus is described in some detail. Typical results are given for densities as low as $10^{13}$ electrons $/ \mathrm{cm}^{3}$.

The magnetoionic theory at hydromagnetic frequencies, S. R. Seshadri, J. Atmospheric Terrest. Phys. 27, No. 5, 617-634 (May 1965).

The characteristics of a plane wave propagating in a two component lossless plasma are studied using the approach of the magnetoionic theory for all directions of propagation with respect to that of the static magnetic field and for frequencies ranging from hydromagnetic to radio frequencies. The propagation ranges of the two modes are obtained by a variant of the well-known CMA diagram and the advantages of this new representation are outlined. In the close neighborhood of the directions parallel and perpendicular to the static magnetic field, the dispersion is found to vary rapidly. The electron gyromagnetic and the plasma resonances as well as the cutoff frequency of the high frequency branch of the ordinary electromagnetic mode show discontinuous variations in the close neighborhood of the direction of the static magnetic field. An interesting result of this investigation is that the so-called ion cyclotron resonance frequency is practically independent of the direction of propagation except in the very close neighborhood of the direction perpendicular to that of the static magnetic field. Frequency and spatial dispersion relations are presented for some typical parameters.

Eigenvalues of a microwave-cavity-lossy-plasma system, J. L. Shohet and C. Moskowitz, J. Appl. Phys. 36, No. 5, 1756-1759 (May 1965).

A cylindrical microwave cavity containing a concentric plasma column can be resonated in an infinite number of electromagnetic wave modes. The resonances all shift upward towards coaxial cavity modes as the electron density in the plasma is increased if collisions are neglected. The characteristic eigenvalue equation for all modes including collisional effects is derived and typical graphs for fundamental modes are presented. The solutions of the eigenvalue equation can be explained on the basis of coupled-mode theory. The empty cavity mode and the coaxial (infinite electron density) mode which are originally overcoupled, uncouple from each other for sufficiently large values of electron collision frequency. The imaginary part of the roots, which is inversely proportional to the $Q$ of the plasma cavity system, shows the two modes distinctly. 
Comparison of electron contents computed with satellite Faraday data from widely spaced stations. G. N. Taylor and R. D. S. Earnshaw, J. Atmospheric Terrest. Phys. 27, No. 4, 525-533 (Apr. 1965).

The analysis of satellite Faraday data by determining the best-fitting linear variation of ionospheric electron content with time is discussed, and a simple test of the validity of the computed results is given. One hundred and ninety-three records of Transit 4A from seven widely spaced stations in Europe have been reduced, and in some cases it has been possible to compare the results obtained from different stations for the same satellite pass. Fairly reliable values of the electron content can be calculated provided that the true variation of Faraday rotation with time has been efficiently sampled and that the true mean variation of electron content is nearly linear. The method appears to work best at middle latitudes, but becomes almost useless near the Auroral zone. Some anomalous electron content gradients have been found.

On the wave propagation in nonuniform media, $\mathrm{H}$. Y. Yee and F. J. Tischer, Proc. IEEE 53, No. 5, 488 (May 1965).

The scattering of plane waves by nonuniform media is currently of considerable interest. Models of this type considered by many authors are confined to simple cases where the permittivity $\epsilon$ is a function of only of one dependent variable, and the permeability $\mu$, is constant. Problems dealt with in several cases are actually second-order homogeneous differential equations. The purpose of this paper is to report that the method of collocation is applicable to achieve an approximate solution. This method has two advantages. 1) There is no limitation on the variation of $\epsilon$ as long as it is a well-behaved function. 2) Good solutions can be achieved even when values of $\epsilon$ are known (by experiment) only at a sufficient number of points in space.

The following publication is available from the Elsevier Publishing Company, New York (publication date, 1965).

Problems of Atmospheric and Space Electricity, 616 pages, edited by S. C. Coroniti.

This publication contains the complete proceedings of the Third International Conference on Atmospheric and Space Electricity held at Montreux, Switzerland, May 5-10, 1963. The meeting was sponsored by I.A.M.A.P., I.A.G.A., and I.U.G.G.

In the preface Dr. Coroniti stated that the theme selected for this conference was "a thorough discussion of the problem areas in atmospheric and space electricity." At the meeting the speakers presented short summaries of their papers, which had previously been distributed to the conferees. These introductory presentations were then followed by, to quote Dr. Coroniti, "vigorous, stimulating and fruitful discussions." The present book is a complete record of all that transpired during these six days in Montreux. Included are the four introductory addresses on the first day, as well as the banquet speech by J. F. Clark on the role of space vehicles in atmospheric electricity.

The specific topics considered at the conference included the following: atmospheric electricity research in various parts of the world, fair weather electricity, action of radioactivity on the parameters of atmospheric electricity, electricity in the terrestrial atmosphere above the exchange layer, generation of electric charges outside thunderstorms, electric forces associated with precipitation, measurements within clouds, charge generation in thunderstorms, physics of lightning, lightning characteristics derived from sferics, lightning protection, ball lightning, whistlers as a phenomenon to study space electricity, electric fields in the ionosphere, space electricity on other planets. As indicated, the scope of the conference is very wide. Also the detail and coverage of the material were extremely diverse. In some cases, only brief or qualitative statements were made, such as in the paper by M. M. Newman on his attempts to study discharge phenomena and VLF propagation by artificially triggered lightning. Fortunately in many other cases, very detailed and quantitative discussions are given, such as found in the comprehensive and well documented paper by P. A. Silberg on ball lightning.

(Review by James R. Wait)
The following publication is available from American Elsevier Publishing Company, 52 Vanderbilt Avenue, New York, N.Y., 10017. The book contains review papers presented at Commission II on Radio and Troposphere During the XIVth General Assembly of URSI, Tokyo, September, 1963.

Progress in Radio Science 1960-1963, Volume II: Radio and Troposphere, edited by Francois du Castel; 291 pages.

Introduction (English and French).

Rapport du Président de la Commission, J. Voge, Centre National d'Etude des Télécommunications, France.

\section{Models of Atmosphere}

Troposphere Models Used in Propagation Theories, H. Bremmer, Philips Research Laboratories, Netherlands.

Modèles D'Atmosphère Découlant D’Observations Météorologiques, P. Misme, Centre National d'Etude des Télécommunications, France.

Models of the Troposphere Derived from Direct Measurements of the Atmospheric Refractive Index, K. Brocks, Hamburg University, Germany.

Models of the Troposphere Derived from Radio-Wave Propagation Experiments, F. Eklund, Research Institute of National Defence, Sweden.

Applications of Turbulence Theory in the Study of Propagation, R. Bolgiano, Jr., Cornell University, U.S.A.

\section{Radioclimatology}

Influences Climatiques en Propagation Troposphérique, F. du Castel, Centre National d'Études des Télécommunications, France.

Climatic Effects in Tropospheric Propagation: Radiometeorological Parameters, J. A. Saxton, Department of Scientific and Industrial Research, United Kingdom.

Radiometeorological Effects in Propagation Over Sea and Islands, F. Ikegami, Electrical Communications Laboratory, Japan.

\section{Influence of Irregular Terrain and Vegetation on Propagation}

Influence of Irregular Terrain on Propagation, J. W. Herbstreit, National Bureau of Standards, U.S.A.

Experimental Results on the Influence of Ground and Vegetation on the Propagation of Electromagnetic Waves, J. Grosskopf, Fernmeldetechnisches Zentralamt, West Germany.

\section{Tropospheric Propagation Affecting Space Communication}

Phenomena of Absorption and Noise of Atmospheric Origin in EarthSpace Communication, D. C. Hogg, Bell Telephone Laboratories, U.S.A.

Effects of Tropospheric Refraction in Earth-Space Links, K. A. Norton, National Bureau of Standards, U.S.A.

\section{Radiometeorology and Cloud Physics}

Progress in Radar Meteorology and Cloud Physics, D. Atlas, Air Force Cambridge Research Laboratories, U.S.A.

Application of Radar to Precipitation Physics and Synoptic Meteorology in Japan, Ichiro Imai, Meteorological Research Institute, Japan.

Developments in Instrumentation for Weather Radar, N. Kodaira, Meteorological Research Institute, Japan.

\section{Propagation of Millemetre and Submillimetre Waves}

Propagation Characteristics of Millimetre Radio Waves, A. W. Straiton, University of Texas, U.S.A.

The following publication is available from American Elsevier Publishing Company, 52 Vanderbilt Avenue, New York, N.Y., 10017. The book contains review papers presented at Commission IV on Radio Noise of Terrestrial Origin during the XIVth General Assembly of URSI, Tokyo, September, 1963.

Progress in Radio Science 1960-1963, Volume IV: Radio Noise of Terrestrial Origin, edited by F. Horner; 133 pages. 
Contents

Report of the Chairman of Commission IV (Radio Noise of Terrestrial Origin), R. A. Helliwell, Stanford University, Stanford, California (U.S.A.).

\section{SESSION ON PROPERTIES OF THE LIGHTNING FLASH SOURCE}

Lightning Flashes and Atmospherics, A. Kimpara, Faculty of Engineering, Nagoya University (Japan).

A Possible Lightning Mechanism as Origin of an ELF Radio Wave, H. Ishikawa, Research Institute of Atmospherics, Nagoya University (Japan).

Sudden Enhancements of Atmospherics on Frequencies Between 10 and $30 \mathrm{kc} / \mathrm{s}$, T. Kamada, Geophysical Institute, University of Tokyo (Japan).

\section{SESSIONS ON IGY RESULTS AND IQSY PROGRAMMES}

Review of IGY Results and IQSY Programme on Atmospheric Noise, F. Horner, D.S.I.R. Radio Research Station, Slough, Bucks (U.K.).

Daily Variations of Atmospheric Noise Level at VLF, E. A. Lauter Observatorium für Ionosphärenforschung, Kühlungsborn (Germany). Frequency Variations of Atmospheric Radio Noise Intensity at Low Frequencies on the Ocean and on Land, Y. I. Likhter and G. I. Terina Izmiran, Moscow (U.S.S.R.).

Some Experimental Results From Lightning Flash Counters, D. Müller-Hillebrand, ${ }^{1}$ Uppsala University (Sweden).

\section{SESSION ON WHISTLERS}

Whistlers, G. McK. Allcock, Physics and Engineering Laboratory, Lower Hutt (New Zealand).

\section{SESSION ON VLF AND NOISE PHENOMENA}

Very Low Frequency Noise from the Terrestrial Magnetosphere, G. R. A. Ellis, Department of Physics, University of Tasmania, Hobart, Tas. (Australia).

Micropulsations of the Earth's Electromagnetic Field in the Frequency Range $0.1-10 \mathrm{c} / \mathrm{s}, \mathrm{J}$. A. Jacobs and T. Watanabe, Institute of Earth Sciences, University of British Columbia, Vancouver (Canada).

A Review of Theoretical Studies on VLF Emissions, I. Kimura, Faculty of Engineering, Kyoto University (Japan).

Les Micropulsationș Magnétiques de Faible Période, R. Gendrin, Centre National d'Études des Télécommunications, Issy-les-Mouliseaux, Seine (France).

\section{SESSION ON GUIDED WAVES IN THE TROPOSPHERE AND} THE IONOSPHERE

Guided Waves in the Earth-Ionosphere Cavity, James R. Wait, Central Radio Propagation Laboratory, National Bureau of Standards, Boulder, Colorado (U.S.A.).

Guidance and Beaming in the Magnetosphere at Hydromagnetic, Audio and Radio Frequencies, H. G. Booker, Stanford Research Institute, California (U.S.A.).

The following publication is available from American Elsevier Publishing Company, 52 Vanderbilt Avenue, New York, N.Y., 10017. The book contains review papers presented at Commission VII on Radioelectronics during the XIVth General Assembly of URSI, Tokyo, September, 1963.

Progress in Radio Science 1960-1963, Volume VII: Radioelectronics, edited by R. E. Burgess; 168 pages.

Activities of URSI Commission VII on Radioelectronics During the Triennium 1960-1963, W. G. Shepherd, Chairman of the Commission.
SESSION ON PLASMAS - GEOPHYSICAL AND ASTROPHYSICAL SCALE

Plasmas-Geophysical and Astrophysical Scale, Reimar Lüst, Max-Planck-Institut für Physik und Astrophysik, München (Germany).

Deterioration of Solar Cells Under High-Energy Electron Bombardment, Osamu Harashima, Yokichi Kano, Hiroshi Tsuru, Ken-Ichi Takahashi, and Hideo Ono, Nippon Electric Co., Kawasaki (Japan).

The Propagation of Storm Plasma in Interplanetary Space, C. P. Sonett, Space Sciences Div., NASA Arms Research Center, Mountain View, Calif. (U.S.A.).

Geomagnetic Storms, C. O. Hines, Department of Geophysical Sciences, University of Chicago, Ill. (U.S.A.).

Interaction of the Solar Wind and the Magnetosphere, A. J. Dessler, Space Science Dept., Rice University. Houston, Texas (U.S.A.).

Ionospheric Disturbances Induced by Space Vehicles. Martti E. Tiuri, Institute of Technology, Helsinki (Finland).

Résumé de la Séance sur Les Plasmas en Géophysique et en Astrophysique, Président: B. Lehnert (Suède).

\section{SESSION ON PLASMAS - LABORATORY SCALE}

Plasmas - Laboratory Scale, Bo Lehnert, Royal Institute of Technology, Stockholm.(Sweden).

Transport Processes in Plasmas, W. B. Thompson, Culham Laboratories, Culham (U.K.).

Thermal and Non-Thermal Emission from Plasmas, G. Bekefi, Department of Physics, Massachusetts Institute of Technology, Cambridge, Mass. (U.S.A.).

Probe Techniques for the Study of Plasma Phenomena, G. Medicus, Electronic Research Branch, Wright-Patterson Air Force Base, Ohio (U.S.A.).

Research Activities on Plasmas in Japan, T. Sekiguchi, Department of Electrical Engineering, University of Tokyo (Japan).

Résumé de la Séance sur Les Plasmas à L'Echelle de Laboratoire, Président: M. P. Bachinski (Canada).

\section{SESSION ON PHYSICS OF MASERS AND LASERS}

Optical Maser Principles, A. Javan, Physics Department M.I.T., Cambridge 39, Mass. (U.S.A.).

Some Properties of a Small Gas Laser, J. Haisma, Phillips Research Laboratories, Eindhoven (Netherlands).

Semi-Classical Theory and Quantum Theory of Photon Noise, Hidetosi Takahasi, Department of Physics, University of Tokyo (Japan).

Semiconductor Injection Masers, R. H. Rediker, Lincoln Laboratory, M.I.T., Lexington, Mass. (U.S.A.).

The Atomic Hydrogen Maser, Norman F. Ramsey, Lyman Physics Laboratory, Harvard University, Cambridge, Mass. (U.S.A.).

Résumé de la Séance sur La Physique des Masers et des Lasers, Président: K. Shimoda (Japan).

\section{SESSION ON APPLICATIONS OF MASERS AND LASERS}

On the Applications of Microwave and Optical Masers, H. E. D. Scovil, Bell Telephone Laboratories, Murray Hill, N.J. (U.S.A.). Recent Developments of Laser Modulation and Demodulation in Japan, Tadakuni Fujii, Nippon Electric Co., Kawasaki (Japan).

Beam Noise Measurement by Laser, S. Saito and Y. Fujii, Institute of Industrial Science, University of Tokyo (Japan).

Non-Linear Optical Effects, R. W. Hellwarth, Hughes Research Laboratory, Stanford University, Calif. (U.S.A.).

Résumé de la Séance sur L'Application des Masers et des Lasers, Président: B. M. Oliver (U.S.A.).

${ }^{1}$ Deceased. 
Transmission loss predictions for tropospheric communication circuits, P. L. Rice, A. G. Longley, K. A. Norton, and A. P. Barsis, NBS Tech. Note 101, Vols. I and II (May 7, 1965)\$1.00 each. This two-part report presents comprehensive methods for predicting cumulative distributions of transmission loss for a wide range of radio frequencies over any type of terrain and in several climatic regions. Such quantitative estimates of propagation characteristics help to determine how well proposed radio systems will meet requirements for satisfactory service, free from harmful interference. Thus they should provide an important step toward more efficient use of the radio frequency spectrum.

The need for comprehensive and accurate prediction methods is clearly demonstrated when measured transmission loss data for a large number of radio paths are shown as a function of path length. In figures I. 1 to I.4 of annex I, long-term median values of attenuation relative to free space for more than 750 radio paths are plotted versus distance. The extremely wide scatter of these data is due mainly to path-to-path differences in terrain profiles and effective antenna heights. Values recorded for a long period of time over a single path show comparable ranges, sometimes exceeding 100 decibels. Such tremendous path-to-path and time variations must be carefully considered, particularly in cases of possible interference between co-channel or adjacent-channel systems.

The detailed point-to-point prediction methods described here depend on propagation path geometry, atmospheric refractivity near the surface of the earth, and specified characteristics of antenna directivity. They have been tested against measurements in the radio frequency range 40 to $10,000 \mathrm{MHz}$ (megacycles per second). Extension of the methods to higher frequencies requires estimates of attenuation due to absorption and scattering of radio energy by various constituents of the atmosphere.

Predictions of long-term median reference values of transmission loss are based on current radio propagation theory. A large sample of radio data was used to develop the empirical predictions of regional, seasonal, and diurnal changes in long-term medians. Estimates of long-term fading relative to observed medians are given for several climatic regions and periods of time, including some regions where few observations are available.

Predictions of transmission loss for paths within the radio horizon are based on geometric-optics ray theory. For paths with a common horizon, Fresnel-Kirchoff knife-edge diffraction theory is applied and extended to predict diffraction attenuation over isolated rounded obstacles. For double horizon paths that extend only slightly beyond the horizon, a modification of the $V$ an der Pol-Bremmer method for computing field intensity in the far diffraction region is used. For longer paths, extending well beyond the radio horizon, predictions are based on forward scatter theory. Radio data were used to estimate the efficiency of scattering at various heights in the atmosphere. Where some doubt exists as to which propagation mechanism predominates, transmission loss is calculated by two methods and the results are combined.

Annex I includes a set of "standard" curves of basic transmission loss and curves showing attenuation below free space for earth space communications, prepared using the methods described in the report. Such curves, and the medians of data shown on figures I.1 to I.4, may serve for general qualitative analysis, but clearly do not take account of particular terrain profiles or climatic effects that may be encountered over a given path.

Annex II supplements the discussion of transmission loss and directive antenna gains given in section 2 . This annex contains a discussion of antenna beam orientation, polarization, and multipath coupling loss.

Annex III contains information required for unusual paths, including exact formulas for computing line-of-sight transmission loss with ground reflections, as well as modifications of the formulas for antenna beams which are elevated, or directed out of the great circle plane. Sample calculations and analytic expressions suitable for use on a digital computer are also included.

Annex IV reviews tropospheric propagation theory with particular attention to the mechanisms of forward scatter from atmospheric turbulence, from layers, or from small randomly oriented surfaces. References to some of the work in this field are included.

Annex V presents a discussion of "phase interference fading" as contrasted to "long-term power fading," provides a method for computing the probability of obtaining adequate service in the presence of noise and/or interfering signals, and includes a brief summary of ways to achieve optimum use of the radio frequency spectrum.

On the continuity equation for electron density in the ionosphere, T. Shimazaki, J. Atmospheric Terrest. Phys. 27, No. 5, 593-604 (May 1965).

The continuity equation for electron density is discussed by considering some representative chemical reactions in the ionospheric regions. Without neglecting the time-dependent and the transport terms, the equation is modified so that the decay term has a form of

$$
\mathscr{L}=\frac{\lambda\left[X_{2}\right]}{N_{e}+\frac{\lambda}{\alpha}\left[X_{2}\right]},
$$

where $\alpha$ and $\lambda$ are the rate coefficients of dissociative recombination and ion-atom interchange reactions, respectively, and the remaining terms are formulated. It is found by rather rough test that these remaining terms are small, and the continuity equation with the decay term of $\mathscr{L}$-type function, which is usually used for the discussion of steady state, may be used for the nonsteady state cases without serious errors. In some cases, however, these remaining terms may be appreciable, and their effects on the continuity equation for electron density may not be neglected. Finally, some detailed formulations for equilibrium electron density are given.

A tabulation of Airy functions, H. T. Dougherty and M. E. Johnson, NBS Tech. Note 228 (Sept. 18, 1964) 20 cents.

A tabulation is provided for Wait's formulation of the Airy function and its first derivative. The argument covers the range of real values from -6.0 to +6.0 in intervals of 0.1 . As an aid for their application the tabulations are also graphed.

Correction of optical distance measurements for the fluctuating atmospheric index of refraction, P. L. Bender and J. C: Owens, J. Geophys. Res. 70, No. 10, 2461-2462 (May 15, 1965). The purpose of this note is to point out that simultaneous measurements of the optical distance over the same path for blue and red light can be used to provide the required correction for the atmospheric index of refraction. The application of this method to interferometric distance measurements has previously been discussed by Erickson.

Nature of the electromagnetic field reflected from a coastline, J. R. Wait, Electronics Letter 1, No. 3, 65-66 (May 1965). In a recent publication, Millar considered the field variation near a coastline when an $H$-polarised plane wave is incident from the sea or land. He stated that disagreement exists between his results and those of Wait. In this communication, it is shown that Millar's conclusions are in error.

On radiation of electromagnetic and electroacoustic waves in plasma, Part II, J. R. W ait, Appl. Sci. Res. Sect. B 12 (1965). The infinitely long cylindrical antenna immersed in a compressible plasma is considered as a boundary-value problem. The analysis starts with a specified voltage at a circumferential gap which is uniformly excited. It is found that the radiation pattern of the antenna is influenced only, to a slight extent, by the finite compressibility of the plasma, provided that the wire diameter is somewhat larger than a Debye length in the plasma. 


\section{Other NBS Publications}

J. Res. 69A (Phys. and Chem.), No. 3 (May-June 1965) 70

cents.

Electronic structure and magnetic properties of the neptunyl ion. J. C. Eisenstein and M. H. L. Pryce.

Heats of transformations in bismuth oxide by differential thermal analysis. E. M. Levin and C. L. McDaniel.

Phase relations between iridium and the sesquioxides in air. S. J. Schneider, J. L. Waring, and R. E. Tressler.

Phase equilibrium relationships in the system $\mathrm{Gd}_{2} \mathrm{O}_{3}-\mathrm{TiO}_{2}$. J. L. Waring and S. J. Schneider.

Acid-base behavior in 50-percent aqueous methanol: thermodynamics of the dissociation of protonated tris(hydroxymethyl) aminomethane and nature of the solvent effect. M. Woodhead, M. Paabo, R. A. Robinson, and R. G. Bates.

Observations of dislocations and surface features in corundum crystals by electron transmission microscopy. D. J. Barber and N. J. Tighe.

Low-frequency dielectric properties of liquid boric oxide. K. H. Stern.

Mass spectrometric study of the effects of nitric oxide and other additives on "purified" active nitrogen. J. T. Herron.

Synthesis and ring structure of 7-acetamido-7-deoxy-L-galactoheptulose. E. J. McDonald.

J. Res. 69A (Phys. and Chem.), No. 4 (July-Aug. 1965), 70 cents.

Correlation of successive atomic steps in crystals by relaxation mode analysis. A. D. Franklin.

Reduction of crystallographic point groups to subgroups by homogeneous stress. H. S. Peiser and J. B. Wachtman; Jr.

Effect of hydrostatic pressure on the refractive indices of some solids. R. M. Waxler and C. E. Weir.

Crystallization kinetics and polymorphic transformations in polybutene-1. J. Powers, J. D. Hoffman, J. J. Weeks, and F. A. Quinn, Jr.

Compliance-time-temperature relationships from indentation measurements on a pure-gum rubber vulcanizate. F. L. Roth, G. W. Bullman, and L. A. Wood.

Distribution function of the end-to-end distances of linear polymers with excluded volume effects. J. Mazur.

An additivity rule for the vapor pressure lowering of aqueous solutions. R. A. Robinson and V. E. Bower.

Franck-Condon factors to high vibrational quantum numbers $\mathrm{V}$ : $\mathrm{O}_{2}$ band systems. R. W. Nicholls.

An absolute temperature scale from $4{ }^{\circ} \mathrm{K}$ to $20^{\circ} \mathrm{K}$ determined from measurements with an acoustical thermometer. H. H. Plumb and G. Cataland

J. Res. 69B (Math. and Math. Phys.), No. 3 (July-Sept.), 75 cents.

A development of the theory of errors with reference to economy of time. M. D. Hersey. Prefatory note by C. Eisenhart.

Transversals and matroid partition. J. Edmonds and D. R. Fulkerson.

Some $L_{2}$ Markoff inequalities. L. F. Shampine.

Some theorems on the permanent. R. A. Brualdi and M. Newman.

On Kirchhoff's law and its generalized application to absorption and emission by cavities. F. J. Kelly.

On a relation between two-dimensional Fourier integrals and series of Hankel transforms. J. V. Cornacchio and R. P. Soni.

On convex metrics. C. Witzgall.

Some extensions of Banach's contraction theorem. P. R. Meyers.

A variant of the two-dimensional Riemann integral. A. J. Goldman.

The use of finite polynomial rings in the factorization of the general polynomial. D. B. Lloyd.

A primal (all-integer) integer programming algorithm. R. D. Young.

J. Res. 69C (Engr. and Instr.), No. 3 (July-Sept. 1965), 75 cents.

Two-terminal dielectric measurements up to $6 \times 10^{8} \mathrm{~Hz}$. M. G. Broadhurst and A. J. Bur.

Improved ten-picofarad fused silica dielectric capacitor. R. D Cutkosky and L. H. Lee.
Errors in the series-parallel buildup of four-terminal resistors. C. H. Page.

Centerable rotator for measuring properties of crystals. C. P. Saylor and H. B. Lowey.

Equipment for single-crystal growth from the melt suitable for substances with a low melting point. A. T. Horton and A. R. Glasgow.

Phase and amplitude contrast microscopy in partially coherent light. M. De and P. K. Mondal.

Exposure time relations for Kossel microdiffraction photographs. H. Yakowitz and D. L. Vieth.

Cartesian diver as a density comparator. H. A. Bowman and R. M. Schoonover.

Cryogenic behavior of selected magnetic materials. J. J. Gniewek and E. Ploge.

Title page, contents, and corrections, Journal of Research 68A, Physics and Chemistry (1964), 5 cents.

Title page, contents, and corrections, Journal of Research 68B, Mathematics and Mathematical Physics (1964), 5 cents.

Title page, contents, and corrections, Journal of Research 68C, Engineering and Instrumentation (1964), 5 cents.

Title page, contents, and corrections, Journal of Research 68D, Radio Science (1964), 15 cents.

Selected tables of atomic spectra. Atomic energy levels and multiplet tables Si II, Si III, Si IV, C. E. Moore, NSRDS-NBS3, Sec. 1 (June 25, 1965), 35 cents.

Electrical engineering units and constants, NBS Misc. Publ. 268 (June 1965), 10 cents, $\$ 6.26$ per 100.

On the effect of heavy ions on LF propagation, with special reference to a nuclear environment, J. R. Johler and L. A. Berry, NBS Tech. 313 (June 7, 1965), 45 cents.

Stability of two-phase annular flow in a vertical pipe, S. Jarvis, Jr., NBS Tech. Note 314 (June 7, 1965), 55 cents.

\section{Chemistry:}

Determination of the composition of complexes and their instability constant by calorimetry, I. The cuprocyanide complexes, A. Brenner, J. Electrochem. Soc. 112, No. 6, 611-621 (June 1965). Emission spectroscopy, B. F. Scribner and M. Margoshes, Treatise on Analytical Chemistry, ed. I. M. Kolthoff and P. J. Elving, 6, pt. 1, ch. 64, 3347-3461 (Inter-Science Publ. Inc., New York, N.Y., 1965).

Gas chromatographic determination of the moisture content of grain, E. L. Weise, R. W. Burke, and J. K. Taylor (Proc. 1963 Intern. Symp. Humidity and Moisture, Wash., D.C.), Book, Humidity and Moisture, ed. A. Wexler, Vol. IV, Principles and Methods of Measuring Moisture in Liquids and Solids, ch. 1, pp. 3-6 (Reinhold Publ. Corp., New York, N.Y., 1965).

Isotopic fractionation of uranium in sandstone, Powder River Basin, Wyoming, and Slick Rock District, Colorado, J. N. Rosholt, A. P. Butler, E. L. Garner, and W. R. Shields, Econ. Geol. 60, No. 2, 199-213 (Mar.-Apr. 1965)

Mass spectrum of yttrium chloride vapor, J. D. McKinley, J. Chem. Phys. 42, No. 6, 2245-2246 (Mar. 15, 1965).

Method of adjusting force constants and its application to $\mathrm{H}_{2} \mathrm{O}$, $\mathrm{H}_{2} \mathrm{CO}, \mathrm{CH}_{2} \mathrm{Cl}$, and their deuterated molecules, T. Shimanouchi and I. Suzuki, J. Chem. Phys. 42, No. 1, 296-308 (Jan. 1, 1965).

On the twilight sodium emission-2. A theoretical model of sodium abundance, M. Gadsden, Ann. Geophys. 20, No. 4, 383-396 (Uct.Dec. 1964).

Oxidation of asphalt flux with oxides of nitrogen, P. G. Campbell and J. R. Wright, Ind. Engr. Chem. Prod. Res. Develop. 4, No. 2 , 121-128 (June 1965).

Quenching of the triplet state of acetone and biacetyl by azoalkanes, R. E. Rebbert and P. Ausloos, J. Am. Chem. Soc. 87, 1847-1852 (May 5, 1965).

Radiation-induced polymerization and other reactions of $n$-perfluoropentadiene-1,4 at high temperature and pressure, D. W. Brown, J. E. Fearn, and R. E. Lowry, J. Polymer Sci. 3, pt. 2, 1641-1660 (1965). 
Strength of fingernails, R. W. Young, S. B. Newman, and R. J. Capott, J. Invest. Dermatol. 44, No. 5, 358-360 (Dec. 23, 1964).

Vacuum-ultraviolet photolysis of ethane in liquid-nitrogen solution, J. A. Pirog and J. R. McNesby, J. Chem. Phys. 42, No. 7, 24902494 (Apr. 1, 1965).

\section{Engineering and Instrumentation:}

A miniature helium turbo-expander for cryogenic refrigeration systems, M. T. Norton (Proc. XI Intern. Congress Refrigeration, Munich, Germany, Aug. 1963), Prog. Refrig. Sci. Technol., 1, $131-135(1963)$

A simple method for measuring the amount of granular materials in sulfur mortars, C. L. Thompson, Mater. Res. Std. 5, No. 5 , 242-243 (May 1965).

A waveguide noise standard, C. K. S. Miller, W. C. Daywitt, and E. Campbell (Proc. Intern. Measurements Conf.), Acta IMEKO 3, 371-382 (1964)

A standard for extremely low values of spectral irradiance, R. Stair, W. B. Fussell, and W. E. Schneider, Appl. Opt. 4, No. 1, 85-89 (Jan. 1965).

Commercial carbon composition resistors as pressure transducers, C. E. Miller, J. W. Dean, and T. M. Flynn, Rev. Sci. Instr. 36, 231-232 (Feb. 1965)

Human engineering in the design of a console for the comparison of volt boxes, P. H. Lowrie, Jr., 19th Annual Instr. Soc. Am. Conf. Exhibit, Preprint No. 12.1-3-64, pp. 1-8 (Oct. 12-15, 1964).

Maintenance of Marine electronic equipment by personnel not trained in electronic maintenance, G. Shapiro and G. J. Rogers, Proc. Annual Tanker Conf. American Petroleum Institute, pp. A-1-A-18 (June 1965).

Optical instrumentation for the biologist: Microscopy, J. R. MeyerArendt, Appl. Opt. 4, No. 1, 1-9 (Jan. 1965).

Precision detector for complex insertion ratio measuring systems, C. M. Allred and R. A. Lawton, IEEE Trans. Instr. Meas. IM-13, Nos. 2 and 3, 76-81 (June-Sept. 1964).

Stress corrosion cracking of metals, H. L. Logan, Metals Engr. Quart. 5, No. 2, 32-38 (May 1965).

Thermodynamic properties of neon from 25 to $300{ }^{\circ} \mathrm{K}$ between 0.1 and 200 atmospheres, R. D. McCarty and R. B. Stewart, Advances in Thermophysical Properties at Extreme Temperatures and Pressures, pp. 84-97 (Am. Soc. Mech. Engr., New York, N.Y., 1965).

The effect of a fatigue crack on the fatigue strength of an aluminum alloy, J. A. Bennett, Mater. Res. Std. 5, No. 5, 235-239 (May 1965).

The role of cryogencis in the production of high and ultra-high vacuum, L. O. Mullen and M. J. Hiza, Cryogenics 4, No. 6, 387394. (Dec. 1964).

Work function measurements on field emitters with prescribed orientation, J. W. Little, T. E. Madey, and R. Klein, J. Appl. Phys. 36, No. 4, 1491-1492 (Apr. 1965).

\section{Mathematics:}

A method for the computation of the error function of a complex variable, O. N. Strand, Math. Comp. XIX, No. 89, 127-129 (Jan. 1965).

Bounds for class numbers, M. Newman, Proc. Pure Mathematics, VIII. Theory of Numbers, pp. 70-77 (Am. Math. Soc., 1965)

Comment on "The use of transforms to sum array factors," M. T. Ma, IEEE Trans. Ant. Prop. AP-13, No. 1, 174 (Jan. 1965).

Distribution of student's $t$ in samples from a rectangular universe, M. M. Siddiqui, Rev. Intern. Stat. Inst. 32, No. 3, 242-250 (1964).

Mathematical programming models for selection of diets to minimize weighted radionuclide intake, H. J. Bracken, Environmental Health Series: Radiological Health, Public Health Serv. Publ. 999-R-4 (Dept. Health, Education, and Welfare, Wash., D.C., Aug, 1963).

\section{Metrology:}

A comparison between the National Bureau of Standards two-pressure humidity generator and the National Bureau of Standards hygrometer, S. Hasegawa, R. W. Hyland, and S. W. Rhodes (Proc. 1963 Intern. Symp. Humidity and Moisture, Wash., D.C.), Book, Humidity and Moisture, Measurement and Control in Science and Industry, ed. A. Wexler, Vol. 3. Fundamentals and
Standards, ch. 22, pp. 455-459 (Reinhold Publ. Corp., New York, N.Y., 1965).

A pneumatic bridge hygrometer for use as a working humidity standard, L. Greenspan (Proc. 1963 Intern. Symp. Humidity and Moisture, Wash., D.C.), Book, Humidity and Moisture. Measurement and Control in Science and Industry, ed. A. Wexler, Vol. 3. Fundamentals and Standards, ch. 20, pp. 433-443 (Reinhold Publ. Corp., New York, N.Y.. 1965).

Letter to the Editor, C. H. Page, Metrologia 1, No. 2, 73-74 (Apr. 1965).

The evolution of designed experiments, W. J. Youden, Proc. IBM Scientific Computing Symp. Statistics, pp. 59-67 (Westchester County, New York, Sept. 1963).

The international system of units, A. G. McNish, Am. Soc. Quality Control Annual Tech. Conf. Trans., pp. 431-438 (1965).

The NBS standard hygrometer, A. Wexler and R. W. Hyland (Proc. 1963 Intern. Symp. Humidity and Moisture, Wash., D.C.), Book, Humidity and Moisture. Measurement and Control in Science and Industry, ed. A. Wexler, Vol. 3, Fundamentals and Standards, ch. 19, pp. 389-431 (Reinhold Publ. Corp., New York, N.Y., 1965).

\section{Physics:}

Adsorption of polymer molecules at low surface coverage, C. A. J. Hoeve, E. A. DiMarzio, and P. Peyser, J. Chem. Phys. 42, No. 7 , 2558-2563 (Apr. 1, 1965).

Characteristics of a Raman laser excited by an ordinary ruby laser, H. Takuma and D. A. Jennings, Proc. IEEE 53, No. 2, 146-149 (Feb. 1965).

Determination of the probability distribution of the number of secondary electrons, Z. Bay and G. Papp, IEEE Trans. Nuclear Sci. NS-1 1, No. 3, 160 (June 1964); Elec. Commun. 40, No. 1, 134 (1965).

Drift-free Mossbauer spectrometer, F. C. Ruegg, J. J. Spijkerman, and J. R. DeVoe, Rev. Sci. Instr. 36, No. 3, 356-359 (Mar. 1965).

Effects of finite heat capacity on spin-lattice relaxation, R. L. Peterson, Phys. Rev. 137, No. 5A, A1444-A1448 (Mar. 1, 1965).

Elastic constants of cubic lead fluoride at room temperature, J. H Wasilik and M. L. Wheat, J. Appl. Phys. 36, No. 3, pt. 1, 791-793 (Mar. 1965).

Elastic resonances in electron scattering from $\mathrm{He}, \mathrm{Ne}, \mathrm{Ar}, \mathrm{Kr}, \mathrm{Xe}$, and $\mathrm{Hg}$, C. E. Kuyatt, J. A. Simpson, and S. R. Mielczarek, Phys. Rev. 138, No. 2A, A385-A399 (Apr. 19, 1965).

Electron microscopy and diffraction of synthetic corundum crystals. I. Pure aluminum oxide grown by the Verneuil process, D. G. Barber and N. J. Tighe, Phil. Mag. \&1, No. 111, 495-512 (May 1965).

Electronic energy bands in $\mathrm{SrTiO}_{3}$ and related oxide semiconductors, A. H. Kahn and A. J. Leyendecker, Proc. 1964 Intern. Conf Physics of Semiconductors, Paris, pp. 33-39 (Physique des Semiconducteurs, Paris, France, 1964).

Ice nucleation by photolyzed silver iodide, G. Burley, Phil. Mag. 10, 527 (Sept. 1964).

Identification of energy levels of negative ions, U. Fano and J. W. Cooper, Phys. Rev. 138, No. 2A, A400-A402 (Apr. 19, 1965).

Influence of spin paramagnetism on superconductivity, R. A. Kam per, R. S. Collier, and Y. Ohori, Phys. Rev. 137, 1A, A75-A77 (Jan. 4, 1965).

Interpretation of $\mathrm{Ar}^{+}-\mathrm{Ar}$ collisions at $50 \mathrm{keV}, \mathrm{U}$. Fano and W. Lichten, Phys. Rev. Letters 14, No. 16, 627-629 (Apr. 19, 1965).

Longitude distribution of proton flares, C. S. Warwick, Astrophys J. 141, No. 2, 500-504 (Feb. 15, 1965).

Mass-spectrometric study of photoionization, II. $\mathrm{H}_{2}, \mathrm{HD}$, and $\mathrm{D}_{2}$, V. H. Dibeler, R. M. Reese, and M. Krauss, J. Chem. Phys. 42 , No. 6, 2045-2048 (Mar. 15, 1965).

Multiple-band conduction in $n$-type rutile $\left(\mathrm{TiO}_{2}\right), \mathrm{J} . \mathrm{H}$. Becker and W. R. Hosler, Phys. Rev. 137, No. 6A, A1872-A1877 (Mar. 15, 1965).

On the behavior of the compressibility along the critical isotherm, S. Y. Larsen and J. M. H. L. Sengers, Advances in Thermophysical Properties at Extreme Temperatures and Pressures, pp. 74-75 (Am. Soc. Mech. Engr., New York, N.Y., 1965).

On the validity of the Lorentz-Lorenz equation near the critical point, S. Y. Larsen, R. D. Mountain, and R. Zwanzig, J. Chem. Phys. 42, No. 6, 2187-2190 (Mar. 15, 1965). 
Plans for proposed new tables of the thermodynamic properties of moist air, J. C. Davis, T. K. Faison, Jr., and P. R. Achenbach (Proc. 1963 Intern. Symp. Humidity and Moisture, Wash., D.C.), Book, Humidity and Moisture, Measurement and Control in Science and Industry, ed. A. Wexler, Vol. 3. Fundamentals and Standards, ch. 11, pp. 315-320 (Reinhold Publ. Corp., New York, N.Y., 1965).

Optical quenching of photoconductivity near the band edge in CdS

L. Grabner, Phys. Rev. Letters 14, No. 14, 551-554 (Apr. 5, 1965).

Polar modes of lattice vibration and polaron coupling constants in rutile $\left(\mathrm{TiO}_{2}\right)$, D. M. Eagles, J. Phys. Chem. Solids 25, No. 11, 1243-1251 (Nov. 1964).

Polaron coupling constants in $\mathrm{SrTiO}_{3}$, D. M. Eagles, J. Phys. Chem. Solids 26, No. 3, 672 (Mar. 1965).

Pressure dependence of the internal field at the ${ }^{59} \mathrm{Co}$ nucleus in a 99.5\% Ni-0.5\% Co alloy, L. H. Bennett, J. Appl. Phys. 36, No. 3, Pt. 2, 942-943 (Mar. 1965).

Stimulated brillouin scattering in the off-axis resonator. H. Takuma and D. A. Jennings, Appl. Phys. Letters 5, 239-241 (Dec. 1964).

Structure of electrodeposited lead dendrites, F. Ogburn, C. Bechtoldt, J. B. Morris, and A. deKoranyi, J. Electrochem. Soc. 112 , No. 6, 574-577 (June 1965).

SU(6) Clebsch-Gordan coefficients for the product $35 \times 56$, J. C. Carter, J. J. Coyne, and S. Meshkov, Phys. Rev. Letters 14, No. 13, 523-526 (Mar. 29, 1965).

The absolute photometry of the zodical light, L. L. Smith, F. E. Roach. and R. W. Owen. Planet. Space Sci. 13, 207-217 (Pergamon Press Ltd., Northern Ireland, 1965).

The correlation of experimental pressure-density-temperature and specific heat data for parahydrogen, H. M. Roder, L. A. Weber, and R. D. Goodwin (Proc. XI Intern. Congress Refrigeration, Munich, Germany, Aug. 1963), Prog. Refrig. Sci. Technol. 1, 187-191 (1963).

Transition from resonant to non-resonant line shape in microwave absorption, A. Ben-Reuven, Phys. Rev. Letters 14, No. 10, 349351 (Mar. 8, 1965).
X-ray spectrochemical analysis of material: cement and dental alloys, B. L. Bean and B. W. Mulligan, Am. Soc. Testing Mater. Spec. Tech. Publ. 373, Application of Advanced and Nuclear Physics to Testing Materials, pp. 25-45 (June 17, 1965).

\section{Radio Science:}

Measurement and standardization of LF to UHF electrical quantities. Sketch of recent world-wide developments, M. C. Selby, Prog. Radio Sci. 1960-1963, Vol. I. Radio Standards and Measurements, pp. 45-55 (Elsevier Publ. Co., Amsterdam, The Netherlands, 1965).

Radio meteorology, J. W. Herbstreit, Science 147, No. 3653, 76-78 (Jan. 1, 1965).

Stratospheric moisture measurements using infrared spectroscopy, D. M. Gates (Proc. 1963 Intern. Symp. Humidity and Moisture, Wash., D.C.), Book, Humidity and Moisture Measurement and Control in Science and Industry, Vol. 2, Applications, Sec. VI, pp. 446-449 (Reinhold Publ. Corp., New York, N.Y., 1965).

The use of electromagnetic signals emitted from nuclear explosions to study long-range VLF propagation, A. G. Jean and J. R. Wait, J. Geophys. Res. 70, No. 5, 1258-1261 (Mar. 1, 1965).

The uses of oblique ionograms in frequency utilization, K. Davies, ITU Telecommun. J. 31, No. 10, pp. 1-7 (Oct. 1964).

Tropospheric propagation, P. L. Rice and J. W. Herbstreit, Advan. Electron. Electron Phys. 20, 199-259 (1964).

* Publications for which a price is indicated are available by purchase from the Superintendent of Documents, U.S. Government Printing Office, Washington, D.C. 20402 (foreign postage, onefourth additional). Reprints from outside journals and the NBS Journal of Research may often be obtained directly from the authors. 